\title{
OPEN Cell geometry and the cytoskeleton impact the nucleo-cytoplasmic localisation of the SMYD3 methyltransferase
}

\author{
David Pereira ${ }^{1,2}$, Alain Richert ${ }^{1}$, Souhila Medjkane ${ }^{2}$, Sylvie Hénon ${ }^{1 凶} \&$ \\ Jonathan B. Weitzman ${ }^{2 \bowtie}$
}

Mechanical cues from the cellular microenvironment are converted into biochemical signals controlling diverse cell behaviours, including growth and differentiation. But it is still unclear how mechanotransduction ultimately affects nuclear readouts, genome function and transcriptional programs. Key signaling pathways and transcription factors can be activated, and can relocalize to the nucleus, upon mechanosensing. Here, we tested the hypothesis that epigenetic regulators, such as methyltransferase enzymes, might also contribute to mechanotransduction. We found that the SMYD3 lysine methyltransferase is spatially redistributed dependent on cell geometry (cell shape and aspect ratio) in murine myoblasts. Specifically, elongated rectangles were less permissive than square shapes to SMYD3 nuclear accumulation, via reduced nuclear import. Notably, SMYD3 has both nuclear and cytoplasmic substrates. The distribution of SMYD3 in response to cell geometry correlated with cytoplasmic and nuclear lysine tri-methylation (Kme3) levels, but not Kme2. Moreover, drugs targeting cytoskeletal acto-myosin induced nuclear accumulation of Smyd3. We also observed that square vs rectangular geometry impacted the nuclear-cytoplasmic relocalisation of several mechanosensitive proteins, notably YAP/TAZ proteins and the SETDB1 methyltransferase. Thus, mechanical cues from cellular geometric shapes are transduced by a combination of transcription factors and epigenetic regulators shuttling between the cell nucleus and cytoplasm. A mechanosensitive epigenetic machinery could potentially affect differentiation programs and cellular memory.

Mechanosensing is a fundamental property of many living cells. As part of their normal physiological functions, cells in multicellular organisms must respond and adapt to mechanical stimuli such as forces, deformations, geometry and stiffness of the extracellular matrix ${ }^{1-4}$. Conversely, aberrant mechanical responsiveness is often associated with severe diseases, including cardiovascular disorders, myopathies, fibrotic diseases, or cancer metastasis. Cells can sense and respond to changes in rigidity by aligning their shape, cytoskeletal structures, and traction forces ${ }^{4,5}$. For example, the sensing of matrix substrate rigidity and geometrical constraints can direct stem cell lineage specification and differentiation programs $s^{2,6,7}$. In recent years, significant progress has been made in understanding the signaling pathways of mechanotransduction, and the resulting gene expression regulation by the modulation of transcription factors activity. Notable examples include the transcriptional coactivators, YAP/ TAZ $^{8}$, and MRTF- $\mathrm{A}^{9}$, which are translocated to the nucleus upon mechano-sensitive activation of the Hippo and Rho/MRTF pathways, respectively. Despite these advances, less attention has been paid to modifications of the chromatin and the epigenetic machinery by mechanical cues. Epigenetic regulators could modulate the transcriptional activity and gene expression programs in differentiation, offering a long-term temporal memory of transient spatial mechano-signals.

Epigenetic mechanisms regulate gene expression through a series of post-translational modifications ${ }^{10-13}$. Epigenetic enzymes can modify residues in the histone N-terminal tails to impact chromatin structure and regulate transcriptional activity ${ }^{13,14}$. Lysine acetylation and lysine methylation have emerged as key regulatory events in epigenetic control of genome function ${ }^{12,15-17}$. Many enzymes containing a characteristic SET domain

\footnotetext{
${ }^{1}$ Matière et Systèmes Complexes (MSC), UMR 7057 CNRS, Université de Paris, Bâtiment Condorcet, Case 7056, 10 rue Alice Domon et Léonie Duquet, 75205 PARIS cedex 13, France. ${ }^{2}$ Epigenetics and Cell Fate (EDC), UMR 7216 CNRS, Université de Paris, Bâtiment Lamarck, Case 7042, 35 rue Hélène Brion, 75205 PARIS cedex 13, France. ${ }^{\square}$ email: sylvie.henon@u-paris.fr; jonathan.weitzman@u-paris.fr
} 
are linked to methylation of lysine residues in histone tails ${ }^{18}$. The resulting mono-, di- or tri-methylated lysine residues (Kme1, Kme2 or Kme3) can exert either activating or repressive effects on gene expression ${ }^{12,19}$. Despite their initial identification as 'histone methyltransferases', many of these enzymes can also modify non-histone proteins, including transcription factors such as $\mathrm{p} 53$, YAP or $\beta$-catenin ${ }^{20-23}$. Intriguingly, some methyltransferases have been recently linked to cytoplasmic, non-histone substrates, suggesting broader roles in regulating cellular states $^{23-25}$.

SMYD3 is a member of the SMYD (SET and MYND domain-containing proteins) lysine methyltransferases that has been implicated in differentiation and cancer progression ${ }^{24,26-30}$. SMYD3 was initially identified as a histone H3K4 methyltransferase enzyme upregulated in metastatic cancers ${ }^{31}$. However, subsequent studies showed that SMYD3 can also methylate H4K5 and H2A.Z.1, depending on the cellular context ${ }^{32,33}$. Furthermore, SMYD3 has cytoplasmic, non-histone targets that contribute to tumorigenesis; for example, SMYD3 can methylate lysine residues in the cytoplasmic domain of the VEGF receptor in angiogenesis or the MAP3K2 signaling kinase in Ras-dependent oncogenesis ${ }^{24,34}$. Despite these striking examples of differential SMYD3 substrates in the nucleus or the cytoplasm, little is known about what regulates the nuclear-cytoplasmic shuttling of SMYD3. SMYD family proteins have been linked to muscle differentiation in a wide range of studies: for example, we recently showed that SMYD3 is critical for the differentiation program of mammalian muscle differentiation ${ }^{35}$. Furthermore, SMYD3 was linked to cardiac and skeletal muscle development in zebrafish ${ }^{36}$, and cytoplasmic activity of SMYD2 maintains skeletal muscle functions ${ }^{37}$. Thus, muscle differentiation could offer an interesting experimental model to explore a link between cell geometry, mechanical cues and epigenetic SMYD proteins. The differentiation process from myoblasts to differentiated myotubes involves extensive changes in cell morphology $\left(\right.$ notably cell elongation ${ }^{38}$ ), as well as gene expression and epigenetic modifications ${ }^{39,40}$. Muscle cells appear to have both cytoplasmic and nuclear targets for members of the SMYD methyltransferase family: for example, SMYD2 methylates cytoplasmic Hsp90 chaperone in myoblasts to promote the interaction with titin $^{37}$, whereas the deletion of Smyd1 impaired myoblast differentiation by decreased expression of muscle-specific genes ${ }^{41}$.

In this study, we used fibronectin-coated adhesive micropatterns to alter cellular geometry (size, shape, and aspect ratio) of murine myoblasts and to investigate the link between cell shape and contractility and the subcellular localisation of the SMYD3 methyltransferase enzyme. Our findings link geometric and mechanical cues to methyltransferase localization and lysine methylation. We uncovered an important link between the geometric constraints and the actomyosin cytoskeleton of myoblast cell and the regulation of SMYD3 subcellular distribution.

\section{Results}

Cell geometry regulates SMYD3 distribution. The recent discovery that the SMYD3 methyltransferase has both nuclear and cytoplasmic targets that are critical for cell proliferation, migration and differentiation ${ }^{24,30,32-35}$, raised the intriguing question how cellular states might regulate nuclear vs. cytoplasmic partitioning. We investigated whether cell spreading affected SMYD3 subcellular localization, using immunofluorescence of a SMYD3 Flag-tagged construct in C2C12 cells stably expressing SMYD3-HA-Flag fusion protein. C2C12 myoblasts assumed a wide variety of sizes and shapes when plated on substrates coated with fibronectin. We noted that SMYD3 was primarily cytoplasmic in C2C12 cells (Fig. 1a), as previously reported for other methyltransferases, such as the related SMYD2 enzyme ${ }^{37}$. We noted that SMYD3 was visible in the nucleus in cells that were rounded or unspread, but became more cytoplasmic in the more elongated cells (Fig. 1b). Indeed, there was a negative correlation between the cell aspect ratio and the nuclear:cytoplasmic partitioning (Fig. 1b). In order to investigate separately the effects of cell spreading area and shape, we plated C2C12 myoblasts on micropatterns of defined sizes and shapes coated with fibronectin and followed SMYD3 localisation using immunofluorescence of the endogenous SMYD3 (WT C2C12 cells) or an exogenous Flag-tagged construct (C2C12 cells stably expressing SMYD3-HA-Flag fusion protein). Previous reports have linked cell spreading area to nuclear localisation of transcriptional regulators (e.g. YAP/TAZ) ${ }^{8}$. Interestingly, we found that SMYD3 nuclear vs cytoplasmic partitioning was affected by the shape of the micropattern, rather than the pattern area per se. Cells plated on rectangular micropatterns with an aspect ratio of 1:5 and 1:8 exhibited more cytoplasmic SMYD3 compared to cells plated on square micropatterns. We observed similar results whether we measured the exogenous SMYD3-HA-Flag (Fig. 1c,d) or the endogenous SMYD3 protein (Figure S1d,e). Most of our analysis focused on the exogenous SMYD3-HA-Flag because of the specificity and efficiency of the antiFlag antibody, but all experiments with the endogenous protein provided similar conclusions. The total amount of SMYD3 protein (either endogenous SMYD3 or exogenous SMYD3-HA-Flag) was not affected by cell geometry (Figure S2a,d). We calculated the nuclear:cytoplasmic ratio (expressed as the [Mean intensity per nuclear pixel staining]/[Mean intensity per cytoplasmic pixel staining]) over a range of patterns areas $\left(500-1800 \mu \mathrm{m}^{2}\right)$ and aspect ratios: squares (1:1) or rectangles (1:5 or 1:8). We consistently observed lower nuclear staining for SMYD3 on all rectangles, compared to squares (Figs. 1d, S1a,b,e). Again, the same observations were confirmed whether we measured the exogenous SMYD3-HA-Flag (Fig. 1d and Figure S1a,b) or the endogenous SMYD3 (Figure S1e). Furthermore, the nuclear:cytoplasmic ratio showed no significant dependence on the spreading areas, for cells plated on square patterns, as well as on rectangular micropatterns, nor on the total amount of SMYD3 (Figure S2b,c). Control experiments with eGFP protein, which freely diffuses through the nuclear pores $^{42}$, showed that the repartition of a freely diffusing protein is hardly affected by geometry (Fig. 1e and Figure S1c). The difference in the nuclear:cytoplasmic ratio of eGFP between cells plated on square patterns or on rectangle micropatterns $\left(1200 \mu \mathrm{m}^{2}\right.$ in area) was significant, but very small; less than $3 \%$, as compared to about $30 \%$ for SMYD3-HA-Flag and about $65 \%$ for endogenous SMYD3. Thus, the nuclear localisation of SMYD3 appears to be influenced by cell geometry, notably mainly by the aspect ratio of the cell. 
a

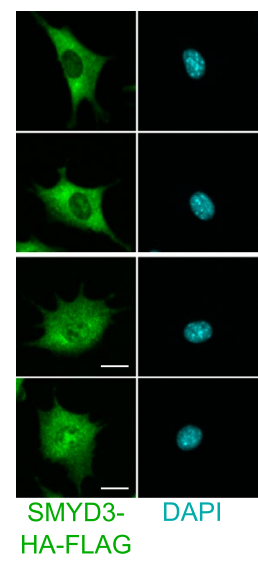

b

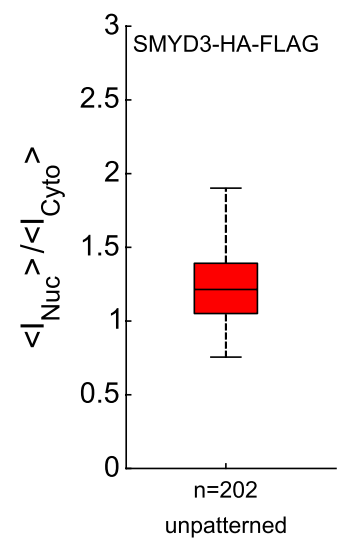

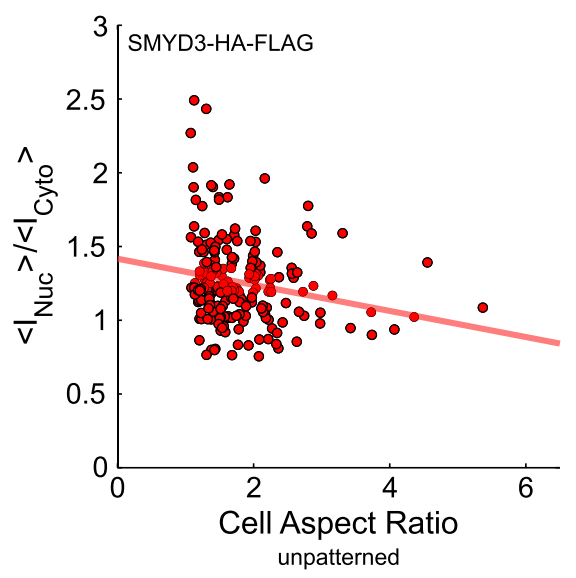

e
C

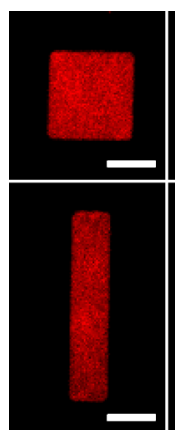

$\mathrm{FN}$

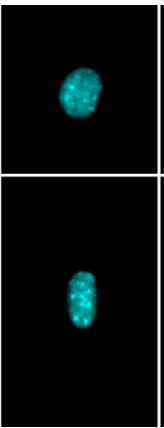

DAPI

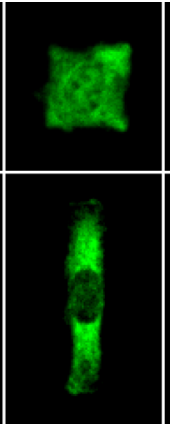

SMYD3HA-FLAG

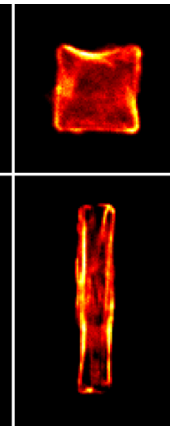

F-ACTIN d

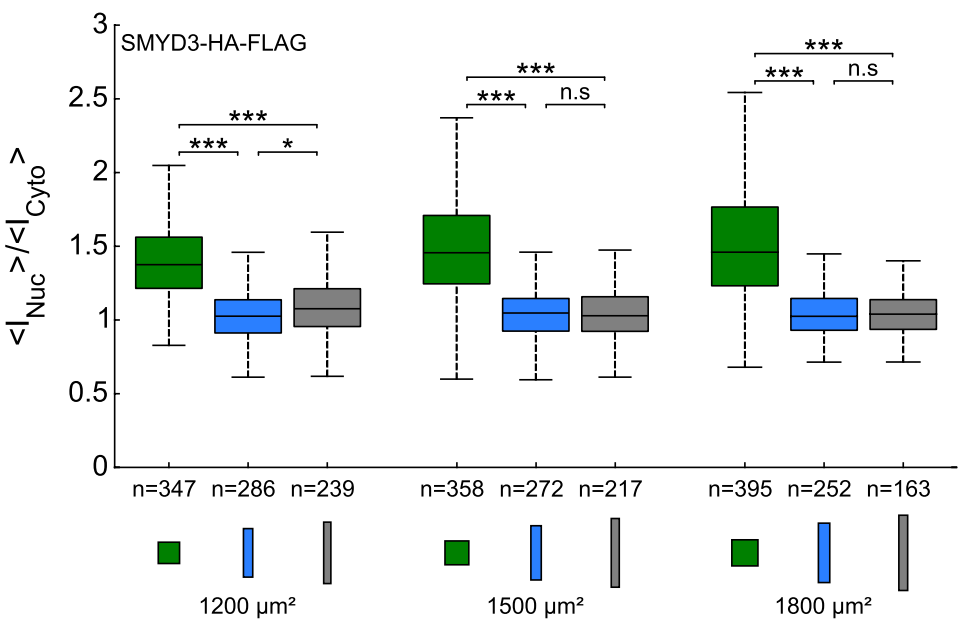

Figure 1. Cell geometry on fibronectin micropatterns regulates SMYD3 distribution. (a) Myoblast C2C12 cells expressing SMYD3-HA-FLAG plated on fibronectin coated substrates. Scale bars: $20 \mu \mathrm{m}$. (b) Quantification of the nuclear:cytoplasmic $\left(\left\langle\mathrm{I}_{\mathrm{Nuc}}\right\rangle\left|<\mathrm{I}_{\text {Cyto }}\right\rangle\right)$ distribution ratio for SMYD3-HA-Flag for cells spread on nonpatterned substrates, and nuclear:cytoplasmic ratio of SMYD3-HA-Flag as a function of cell aspect ratio for cells spread on non-patterned substrates. The red line represents a linear regression $(n=202)$. (c) Myoblast C2C12 cells expressing SMYD3-HA-FLAG were plated on fibronectin micropatterns with the same area $\left(1200 \mu \mathrm{m}^{2}\right)$, but different aspect ratios; square (1:1, upper panel) or rectangle (1:5, lower panel). The micrographs show the fibronectin (FN) patterning, the nuclear DNA staining (DAPI), SMYD3-HA-Flag protein location and F-actin (SiR-actin). The SMYD3 nuclear:cytoplasmic distribution appears different between square and rectangle patterns. Scale bars: $20 \mu \mathrm{m}$. (d) Quantification of the nuclear:cytoplasmic $\left.\left(<\mathrm{I}_{\mathrm{Nuc}}\right\rangle /\left\langle\mathrm{I}_{\text {Cyto }}\right\rangle\right)$ distribution ratio for SMYD3-HA-Flag over a range of pattern areas $\left(1200-1800 \mu \mathrm{m}^{2}\right)$ and geometries: squares $(1: 1$ aspect ratio, green), rectangles (1:5 aspect ratio, blue) and elongated rectangles (1:8 aspect ratio, grey).The median value of the ratio is $27-42 \%$ higher for cells plated on squares than on rectangle patterns. A Kruskal-Wallis test was performed between conditions with identical areas. (e) Quantification of the nuclear:cytoplasmic $\left.\left(<\mathrm{I}_{\mathrm{Nuc}}\right\rangle \mid\left\langle\mathrm{I}_{\mathrm{Cyto}}\right\rangle\right)$ distribution ratio for control eGFP transfected into $\mathrm{C} 2 \mathrm{C} 12$ cells on square or rectangle patterns $\left(1200 \mu \mathrm{m}^{2}\right)$. The eGFP nuclear:cytoplasmic distribution is slightly different (less than $3 \%$ ) between square and rectangle patterns. A two-sample t-test was performed. $\mathrm{n}=$ number of individual cells measured. ${ }^{\star} p<0.05$, ${ }^{* * *} p<0.001, n . s$. not statistically significant.

Correlating SMYD3 cellular distribution with lysine methylation. The SMYD3 methyltransferase has a number of reported substrates, depending on the cell type and cellular state. These include nuclear histone substrates (e.g. histone H3K4, H4K5, and H2A.Z.1) ${ }^{31-33}$, cytoplasmic proteins (e.g. VEGFR1 receptor and the

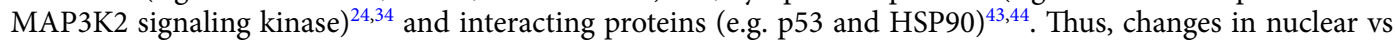
cytoplasmic distribution could likely affect SMYD3 protein interactions and substrate methylation patterns. To investigate whether changes in SMYD3 localisation correlated with lysine methylation, we performed experiments with antibodies recognizing tri-methylated (Kme3) or bi-methylated (Kme2) lysine. We used these panmethyl-lysine tools to catch all potential SMYD3 targets. We found a strong correlation between the SMYD3HA-Flag localisation and Kme3 staining, in terms of nuclear:cytoplasmic ratios (Fig. 2a). Furthermore, image analysis suggested a co-localisation of SMYD3 and Kme3 staining (Fig. 2b), which was quantitatively confirmed 
a

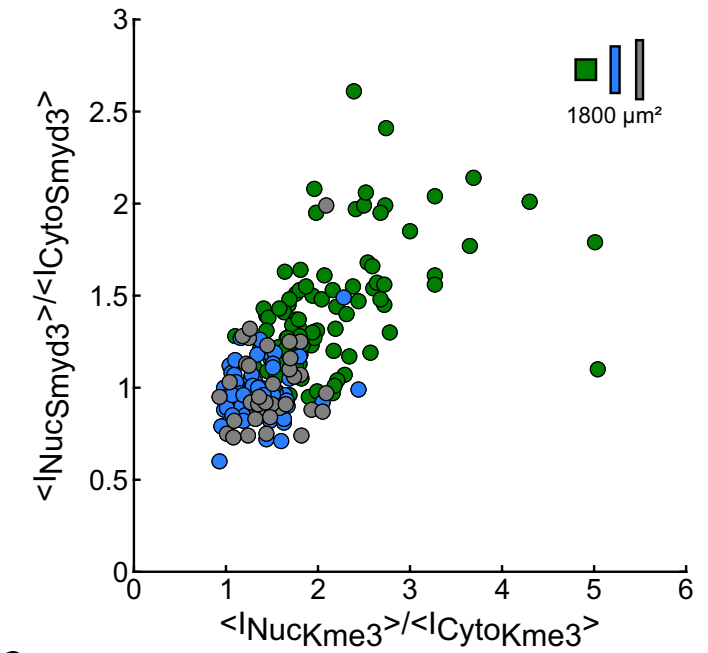

C
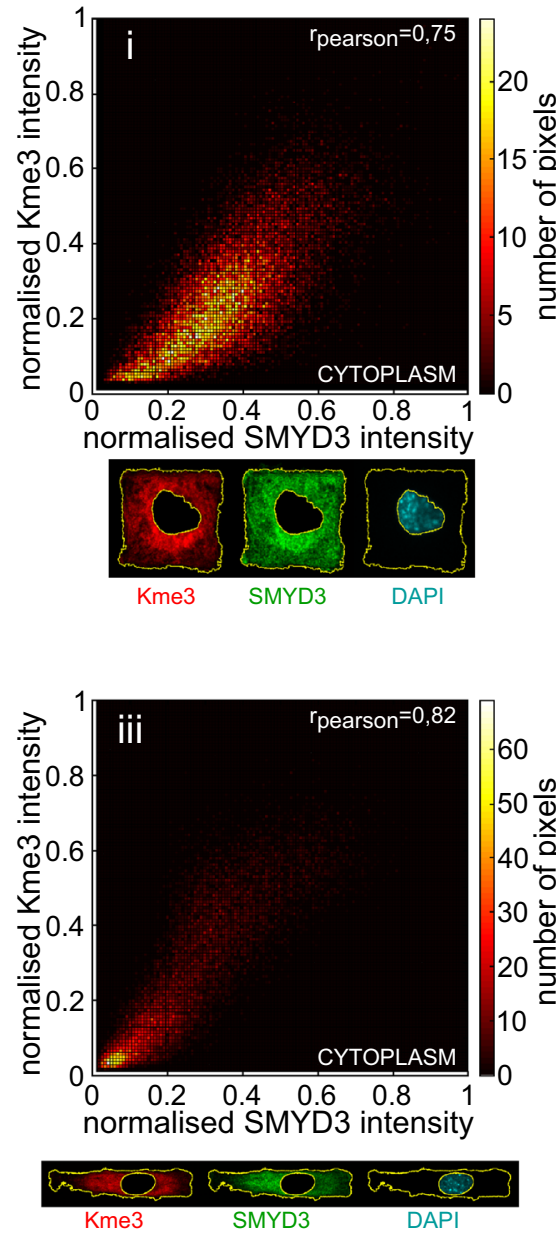

b

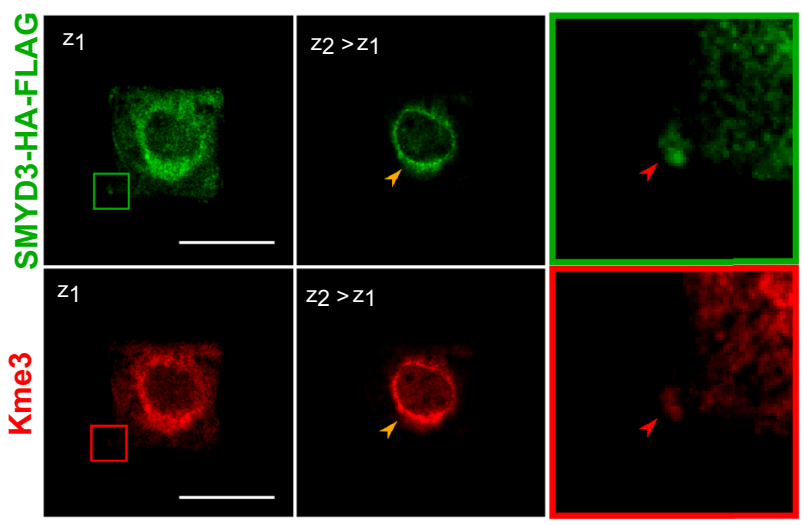

d
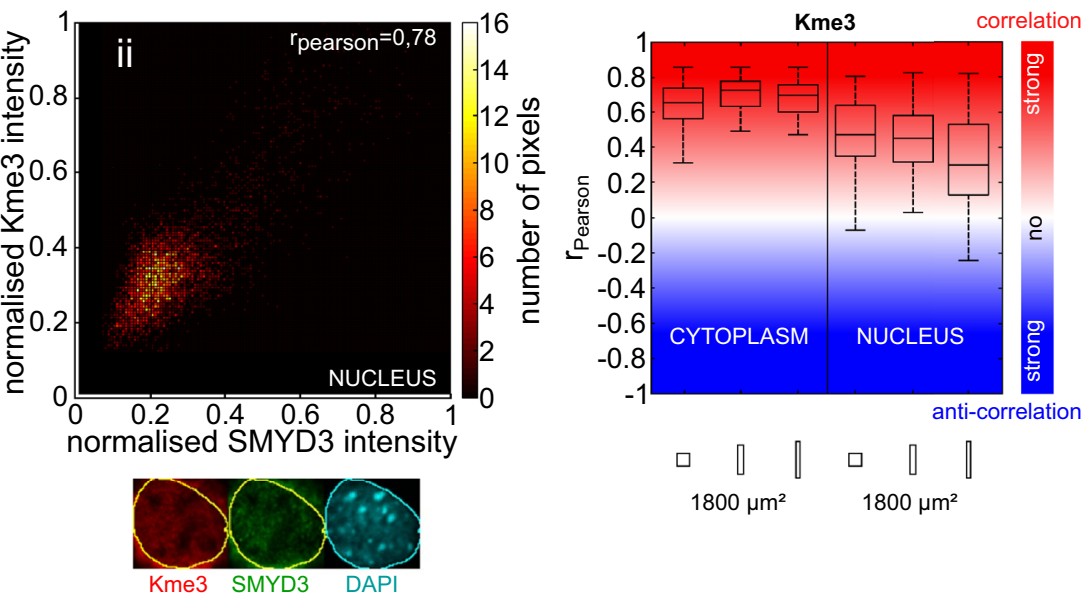

e
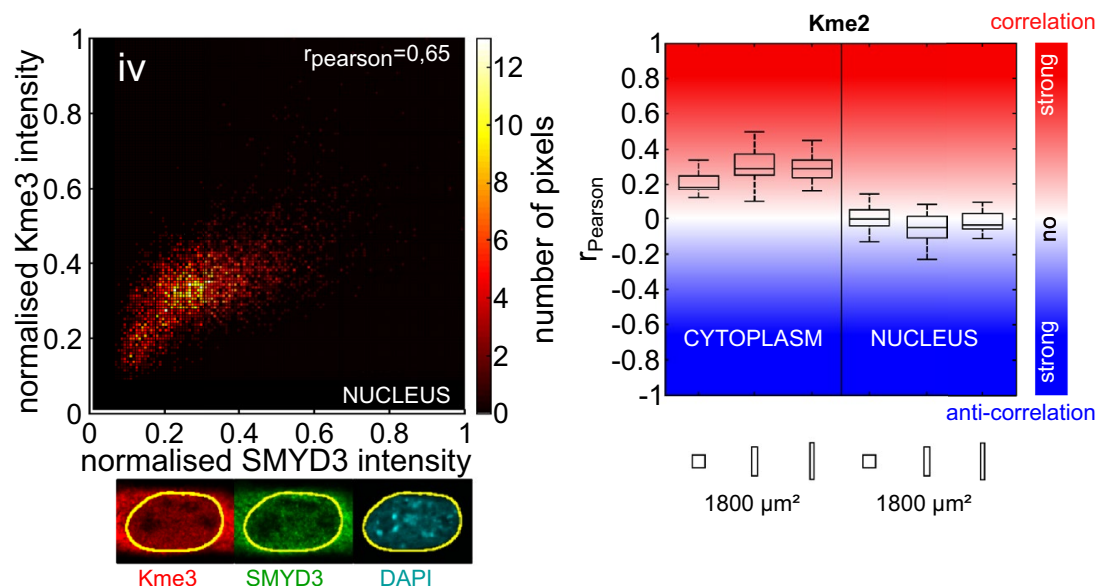

Figure 2. SMYD3 cellular distribution correlates with the lysine trimethylation (Kme3). (a) The increased nuclear distribution of SMYD3-HA-FLAG on square patterns (green dots; $n=105$ ) correlates with higher nuclear staining for lysine tri-methylation marks (Kme3). Conversely, rectangle patterns (blue and grey spots; $n=68$ and 37, respectively) have higher SMYD3 and Kme3 levels in the cytoplasm. (b) Confocal microscopy images of a C2C12 cell spread on a square micropattern, showing the co-localisation of SMYD3HA-FLAG (green) and lysine tri-methylation Kme3 (red) marks. The magnified square highlights SMYD3/Kme3 colocalisation. Scale bars: $30 \mu \mathrm{m}$. (c) Upper panels: Detailed representation of the SMYD3 and Kme3 co-localisation within (i) the cytoplasm and (ii) the nucleus for a cell spread on a square micropattern. Lower panels: the same quantification for a cell spread on a rectangle micropattern showing cytoplasmic (iii) and nuclear (iv) quantification. (d) Graphical representation of the correlation (Pearson coefficient) between SMYD3 and Kme3 lysine tri-methylation localisation. $n=$ numbers of individual cells measured: square $n=105$, rectangle 1:5 $n=68$, rectangle 1:8=37. (e) Graphical representation showing a quantified lack of correlation (Pearson coefficient) between SMYD3 and Kme2 lysine di-methylation localization. $n=$ numbers of individual cells measured: square $n=24$, rectangle $1: 5 n=18$, rectangle $1: 8 n=19$. 
by a high value of the Pearson correlation coefficient, both for cells spread on square micropatterns and on rectangular micropatterns, and both for nuclear and cytoplasmic staining, yet with a better correlation for the cytoplasm (Fig. 2c,d). This correlation was restricted to tri-methylated lysine residues; we failed to observe a correlation when Kme2 antibodies were tested (Fig. 2e); neither in the nucleus (Pearson coefficient $\sim 0$ ), nor in the cytoplasm (Pearson coefficient $<0.3$ ). Thus, the effect of cell geometry on SMYD3 localisation appeared to directly correlate with lysine tri-methylation in both the nucleus and the cytoplasm.

The dynamics of SMYD3 nucleo-cytoplasmic shuttling and the role of the cytoskeleton. Nothing is known about the mechanisms underlying SMYD3 localisation and we failed to identify a clear nuclear localization signal (NLS) or nuclear export signal (NES). To investigate nucleo-cytoplasmic shuttling mechanisms, we treated C2C12 cells with Leptomycin B (LMB). Low LMB concentrations bind to CRM1/exportin 1 and block the nuclear export of many proteins ${ }^{45-47}$. We found that LMB treatment led to nuclear accumulation of SMYD3-HA-Flag in C2C12 cells (Fig. 3a,b). Although this LMB-induced nuclear accumulation was observed for all the cells, it was most marked in cells with a low aspect ratio (Fig. 3c). For cells plated on micropatterns (Fig. 3e) the increase in the nuclear:cytoplasmic ratio was $~ 30 \%$ on squares vs $20 \%$ on rectangles. To investigate the dynamics of the SMYD3 redistribution, we performed laser bleaching experiments on the cell nucleus or the cytoplasm of cells transfected with SMYD3-eGFP and used fluorescence recovery after photobleaching (FRAP) analysis to follow the nuclear recovery timing (Fig. 3f-i). FRAP analysis experiments showed that the nuclear export of SMYD3-eGFP is faster than its import, which is in agreement with a mainly cytoplasmic localisation. Furthermore, the cell geometry (square vs rectangular micropatterns) impacted the speed of nuclear import, rather than the nuclear export dynamics (Fig. 3h,i). The recovery rate of SMYD3 in the nucleus was significantly higher in square patterns compared to rectangles (Fig. 3h, Figure S3a), whereas the nuclear decay rates after cytoplasm photobleaching were similar, independent of the geometry (Fig. 3i, Figure S3b). These drugtreatment and photobleaching experiments clearly showed that nuclear import of SMYD3 is impacted by cell geometry; i.e. relocalisation is faster for cells plated on square patterns than for cells on rectangles.

It is well-established that the actin cytoskeleton plays a major role in mechano-sensing phenomena. We therefore tested whether cell geometry impacted the amount of F-actin leading to different nuclear localization of SMYD3 (Figure S4). We did not find any correlation between cell shape and the total amount of F-actin, nor between SMYD3 nuclear:cytoplasmic ratios and the amount of F-actin (Figure S4a-d). On the contrary the network of F-actin stress fibers of cells on rectangular micropatterns vs square micropatterns were qualitatively different (Figure S5): all the stress fibers almost aligned along the long axis for cells on rectangles while there was no preferred direction for cells on squares ${ }^{48}$, furthermore several apical stress fibers of the so-called actin-cap ${ }^{49}$ indented the nucleus of all the cells on rectangle ${ }^{50}$ while it was never observed for cells on squares. Finally, we treated $\mathrm{C} 2 \mathrm{C} 12$ cells with drugs that disrupt the cytoskeleton or impair acto-myosin contractility and measured the effect on SMYD3 distribution. We treated cells with Blebbistatin (that inhibits acto-myosin contractility) or Y-27632 (a pharmacological inhibitor of the Rho-associated protein kinase, ROCK signaling pathway) (Fig. 4). We also treated cells with the Latrunculin A or Latrunculin B inhibitors which disrupt actin filaments. In all cases, perturbing the cellular cytoskeletal integrity increased the nuclear localization of SMYD3-HA-Flag (Fig. 4b), on both square and rectangular micropatterns, as well as non-patterned substrates (Fig. 4a-c). Thus, the import of SMYD3 into the nucleus in response to mechano-sensing signals is regulated by the organization of acto-myosin cytoskeleton and/or its contractility.

Cell geometry impacts the nucleo:cytosplasmic distribution of a range of regulatory factors. We hypothesized that SMYD3 is not the only transcriptional regulator with a dynamic nuclear-cytoplasmic distribution in response to geometric cues. We sought to test whether other reported mechano-sensitive proteins are also impacted by the square vs rectangle cell geometry. We used our experimental set-up to investigate nuclear:cytoplasmic ratios for $\mathrm{C} 2 \mathrm{C} 12$ cells spread on square or rectangular fibronectin patterns over a range of pattern areas (500-1800 $\mu \mathrm{m}^{2}$ ) (Fig. 5 and Figure S6). We tested localization of another methyltransferase, SETDB1, which was also recently reported to be cytoplasmic ${ }^{25}$. We tested G-actin, which is known to play a role in gene transcription ${ }^{51}$. We also tested YAP/TAZ, two paralogue transcriptional regulators that are a paradigm example of mechanical regulation and nucleo-cytoplasmic shuttling. ${ }^{8}$. We observed in all cases that nuclear:cytoplasmic ratios were higher for cells plated on square micropatterns, compared to rectangular patterns (Fig. 5 and Figure S6). Notably, we found that YAP/TAZ nucleo-cytoplasmic repartition was affected by cell size, becoming more nuclear upon spreading on larger and larger areas (Fig. 5a,b, Figure S6a,b). However, we noted that this phenomenon was most remarkable on square patterns, compared to rectangles (aspect ratio 1:5 or 1:8) (Fig. 5b, Figure S6a,b). In contrast, the SETDB1 methyltransferase (Figure S6c) and the G-actin, stained with DNase 1, (Fig. 5c,d and Figure S6d) distributions behaved similarly to the SMYD3 methyltransferase; the nucleo:cytosplasmic ratios did not significantly depend on spreading area per se, but were higher in cells plated on square patterns (in the range $1200-1500 \mu \mathrm{m}^{2}$ ) (Figure S6c). Hence, the cell geometry affects the subcellular localization of a range of different proteins, including transcription regulators and epigenetic modulators.

\section{Discussion}

Our study represents a first step towards placing lysine methylation on the pathway from extracellular mechanotransduction to nuclear outcomes. We hypothesized that lysine methylation and chromatin modifiers could offer a chain in the missing conceptual link between transient mechanical signals that are transduced in response to extracellular rigidity and the long-term maintenance of differentiation programs. Lysine methylation events are versatile (lysine can be modified on three different positions me1, me2 or me3, with different functional consequences) and relatively stable. The finding that SMYD proteins have both nuclear and cytoplasmic substrates 
a

SMYD3-HA FLAG DAPI
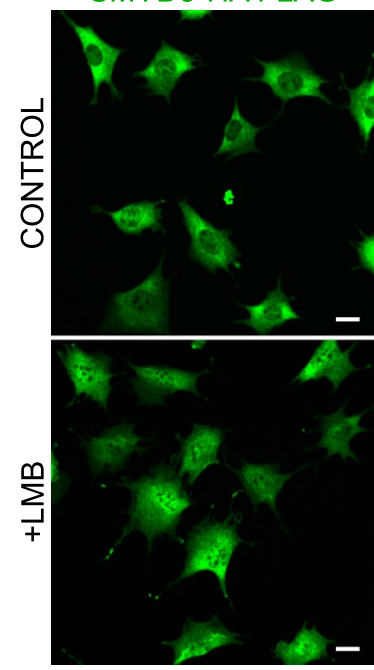

d
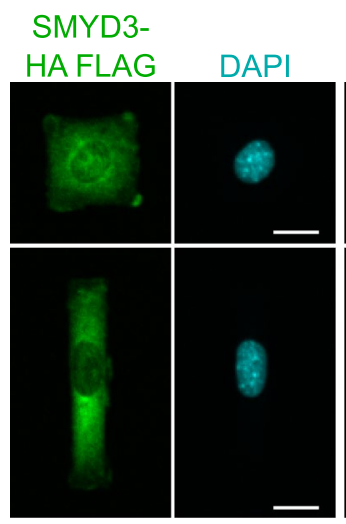

CONTROL

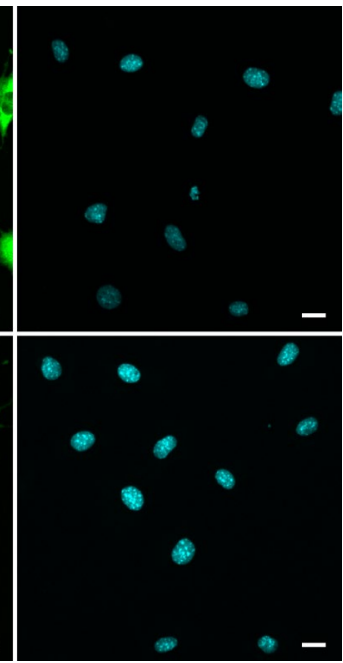

SMYD3-

HAFLAG DAPI

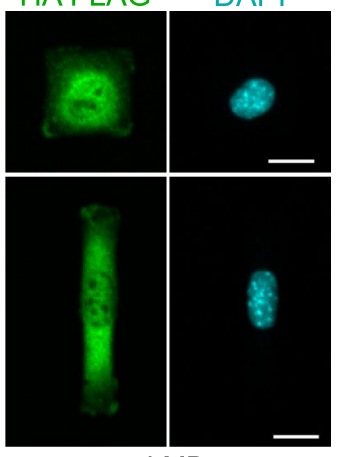

+LMB

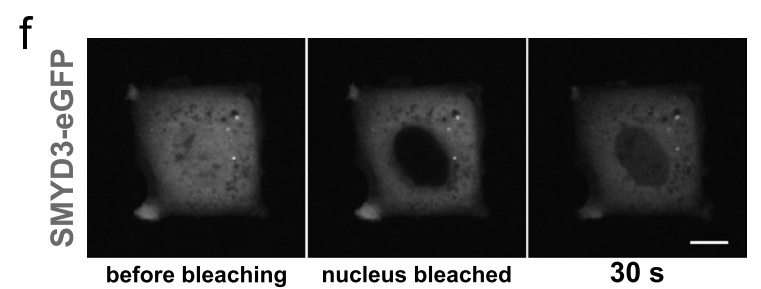

g

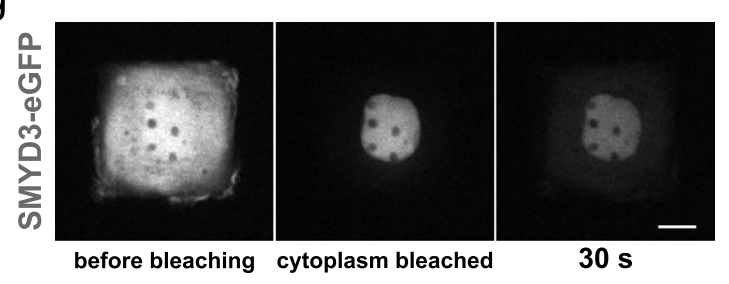

b

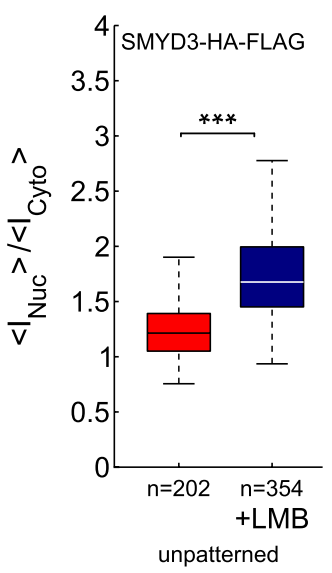

C

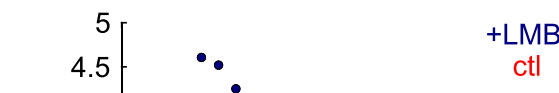

e

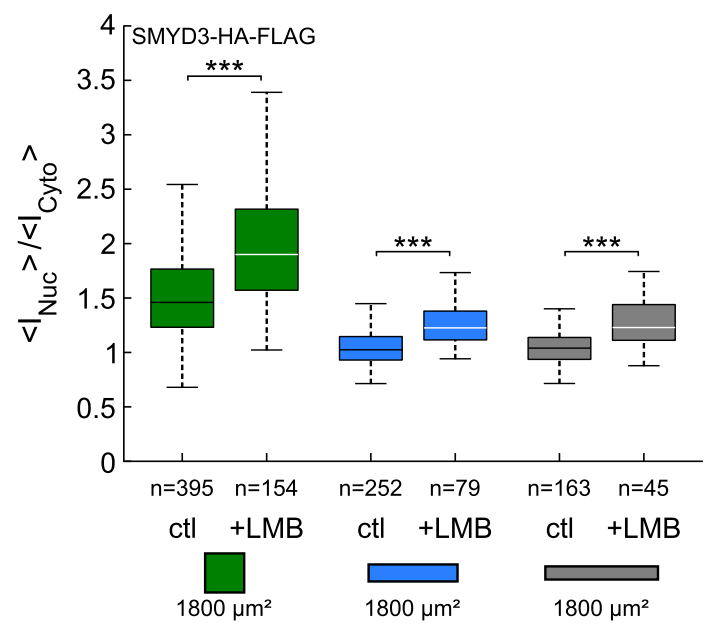

$\mathrm{h}$
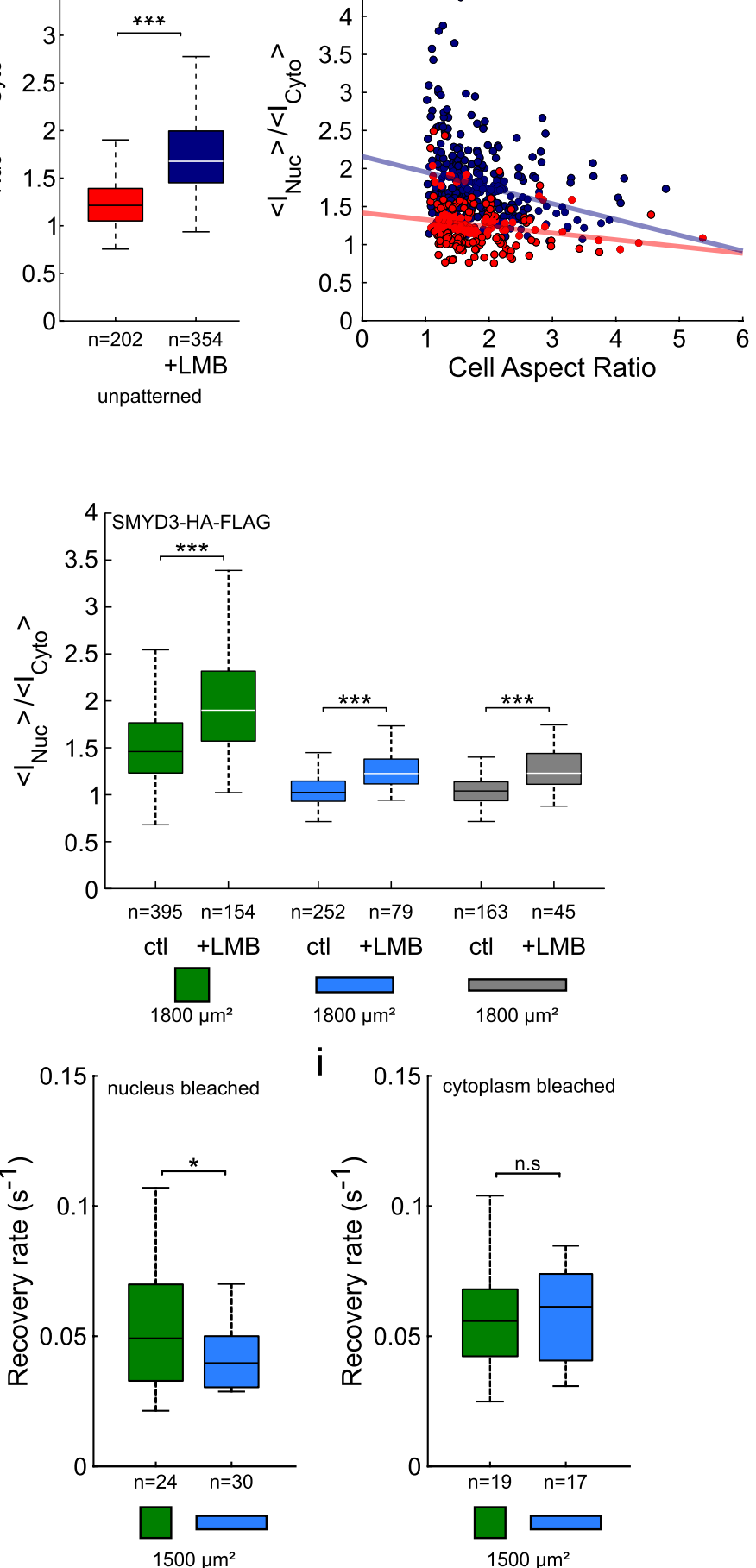

i

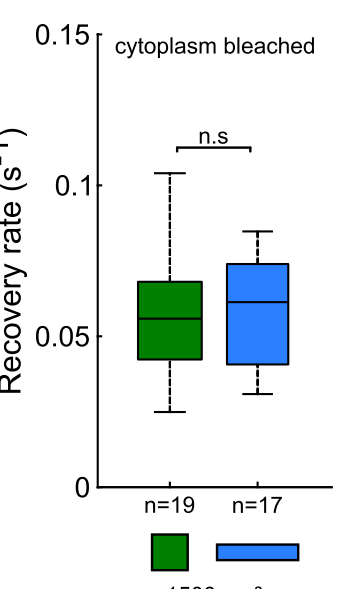

$1500 \mu \mathrm{m}^{2}$ 
४Figure 3. Cell geometry impacts the dynamics of SMYD3 nuclear import. (a) Micrographs of C2C12 cells treated with the nuclear export inhibitor leptomycin B (+LMB) causing increased SMYD3 nuclear localisation. (b) Quantification of the nuclear:cytoplasmic $\left(\left\langle\mathrm{I}_{\mathrm{Nuc}}\right\rangle /\left\langle\mathrm{I}_{\mathrm{Cyto}}\right\rangle\right)$ distribution of SMYD3 in C2C12 cells treated with Leptomycin B (+ LMB, blue) compared to untreated controls (ctl, red). Cells are spread on non-patterned substrates. The two distributions are significantly different (two-sample t-test). (c) Nuclear:cytoplasmic ratio of SMYD3-HA-Flag as a function of cell aspect ratio for cells spread on non-patterned substrates. The red and blue lines are linear regressions. (d) Micrographs of $\mathrm{C} 2 \mathrm{C} 12$ cells treated with the nuclear export inhibitor leptomycin B (+ LMB) causing increased SMYD3 nuclear localization. Cells are plated on square or rectangle $(1: 5$, blue $)$ micropatterns $\left(\right.$ area $\left.=1800 \mu \mathrm{m}^{2}\right)$. (e) Quantification of the nuclear:cytoplasmic $\left(\left\langle\mathrm{I}_{\mathrm{Nuc}}\right\rangle /\left\langle\mathrm{I}_{\mathrm{Cyto}}\right\rangle\right)$ distribution of SMYD3 in C2C12 cells treated with Leptomycin B (+ LMB) compared to untreated controls (ctl) following plating on square (green) or rectangle $(1: 5$, blue) or elongated rectangle $(1: 8$, grey) micropatterns $\left(\right.$ area $\left.=1800 \mu \mathrm{m}^{2}\right)$. A two-sample t-test was performed between conditions with identical aspect ratios. $(\mathbf{f})$ FRAP analysis of SMYD3-eGFP localization, before, just after and $30 \mathrm{~s}$ after nuclear photo-bleaching. (g) FRAP analysis of SMYD3-eGFP localization, before just after and $30 \mathrm{~s}$ after cytoplasmic photo-bleaching. (h) Quantification of the recovery rate of fluorescence intensity after nuclear photo-bleaching of C2C12 cells spread on square (green) or rectangle 1:5 (blue) $1500 \mu^{2}$ micropatterns. A two-sample t-test was performed. (i) Quantification of the decay rate of nuclear fluorescence intensity after cytoplasmic bleaching of cells spread on square (green) or 1:5 rectangle (blue) $1500 \mu \mathrm{m}^{2}$ micropatterns. A Wilcoxon rank sum test was performed. $\mathrm{n}=$ number of individual cells measured. ${ }^{\star} p<0.05,{ }^{* *} p<0.001, n . s$. not statistically significant.

broadens their potential importance in regulating cellular function in physiological and pathological contexts. We propose, for the first time, that the mechano-sensitivity of SMYD3 distribution could change its cellular substrates and subsequent nuclear vs cytoplasmic functions. It will be interesting in the future to explore in more detail which selective SMYD3 substrates, histone or non-histone, are mechano-sensitive and what the functional consequences are.

Many previous studies demonstrated that cell spreading impacts nuclear import of transcriptional regulators (e.g. YAP/TAZ, NFKB) $)^{8,52}$, but rarely have these studies carefully examined the relative impact of shape, size or geometry. Our experimental system of fibronectin-coated micropatterns allowed us to control the effect of shape, size and aspect ratio on the localization of different proteins, including transcription regulators such as YAP/TAZ and epigenetic regulators such as SMYD3 or SETDB1. Notably, we observed that increased size led to nuclear accumulation of YAP/TAZ coactivators, as previously reported (Fig. 5a,b and Figure S6a,b). However, we also observed that the nuclear:cytoplasmic ratio of YAP/TAZ depends on geometry, with a preference to nuclear accumulation on squares as compared to rectangles, as recently reported in $\mathrm{C} 2 \mathrm{C} 12 \mathrm{cells}^{38}$. Furthermore, the increase in nuclear localization with spreading area is much more marked for squares than for rectangles. Intriguingly, the spatial redistribution of SMYD3 showed a relatively low sensitivity to cell spreading area, but a marked sensitivity to cell shape, with preferred cytoplasmic localization for elongated cells (rectangles with an aspect ratio of 1:5 or 1:8) as compared to isotropically shaped cells (squares). These results highlight a novel distinction between square vs rectangular geometries in regulating a wide range of factors and merits further investigation. The preference for cytoplasmic accumulation on rectangular patterns was clearly evident for other factors tested, e.g. YAP/TAZ and the SETDB1/ESET methyltransferase (Fig. 5 and Figure S6) and SMAD proteins (not shown). Notably, cytoplasmic relocalisation of SETDB1 was previously linked to muscle differentiation ${ }^{25}$. Furthermore YAP/TAZ has been shown to regulate proliferation ${ }^{53-55}$. Finally, accumulation of H3K27me3 was observed during mechanically regulated lineage commitment of epidermal progenitor cells ${ }^{56}$. It is thus tempting to speculate that transitions of aspect ratios, for example during the myogenic differentiation from isotropically shaped myoblasts to elongated myocytes and fused myotubes, could induce mechanical signals that affect the nuclear:cytoplasmic partitioning of regulatory factors. SMYD3 is linked to muscle differentiation and appears to play a nuclear transcriptional role at earlier time points in myogenesis ${ }^{35}$. Furthermore, the deacetylase HDAC3 was reported to be differentially activated by different geometries (aspect ratios) in mouse fibroblast ${ }^{57}$. It would be interesting to look for other differentiation scenarios when methyltransferases switch from nuclear to cytoplasmic functions in response to mechanical signaling.

Our experiments with drug treatments (Figs. 3 and 4) suggest that SMYD3 relocalisation is due to mechano-induced import mechanism and that the SMYD3 distribution is regulated by the acto-myosin contractile cytoskeleton. SMYD3 does not have an obvious NLR or NES sequence and further work will be required to define the mechanism of shuttling. An obvious question for future studies is, what are the functional consequences of SMYD3 relocalisation on the methylation of cytoplasmic vs nuclear substrates and gene regulation? There is a debate about what are the most biologically relevant SMYD3 histone targets and few gene targets have been identified. It is likely that that substrates and targets are cell-type specific. We previously reported that SMYD3 regulates Mmp-9 transcription in fibrosarcoma cells ${ }^{27}$. This gene seems not be a SMYD3 target in mouse myoblasts (unpublished data), but it is intriguing that the $M m p-9$ gene is regulated by matrix stiffness and mechanotransduction in other systems ${ }^{58}$. Also, the activation of cytoplasmic targets is determined by the cellular context, making it difficult to directly predict the mechanistic insights from our experimental system. Our experiments with pan-Lysine-me3 antibodies support a correlation between localization and methyltransferase activity, especially in the cytoplasm, where the correlation is even better than in the nucleus. The relocalisation of SETDB1/ESET has been linked to release from genomic targets and to contribute to muscle differentiation ${ }^{25}$. We favour a model in which specific cell geometries affect nuclear vs cytoplasmic repartitioning of SMYD3 (and other methyltransferases) leading to shifts in substrate encounters and regulation by methylation. 


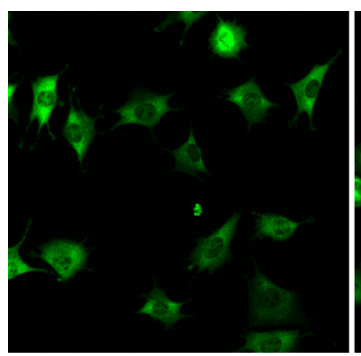

Control

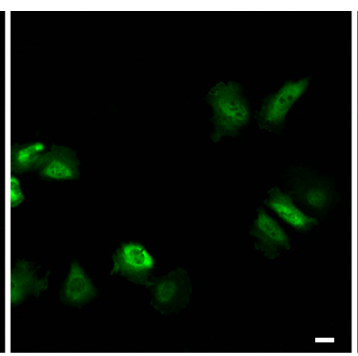

Latrunculin A

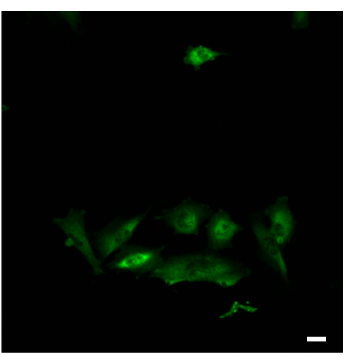

Latrunculin B

C

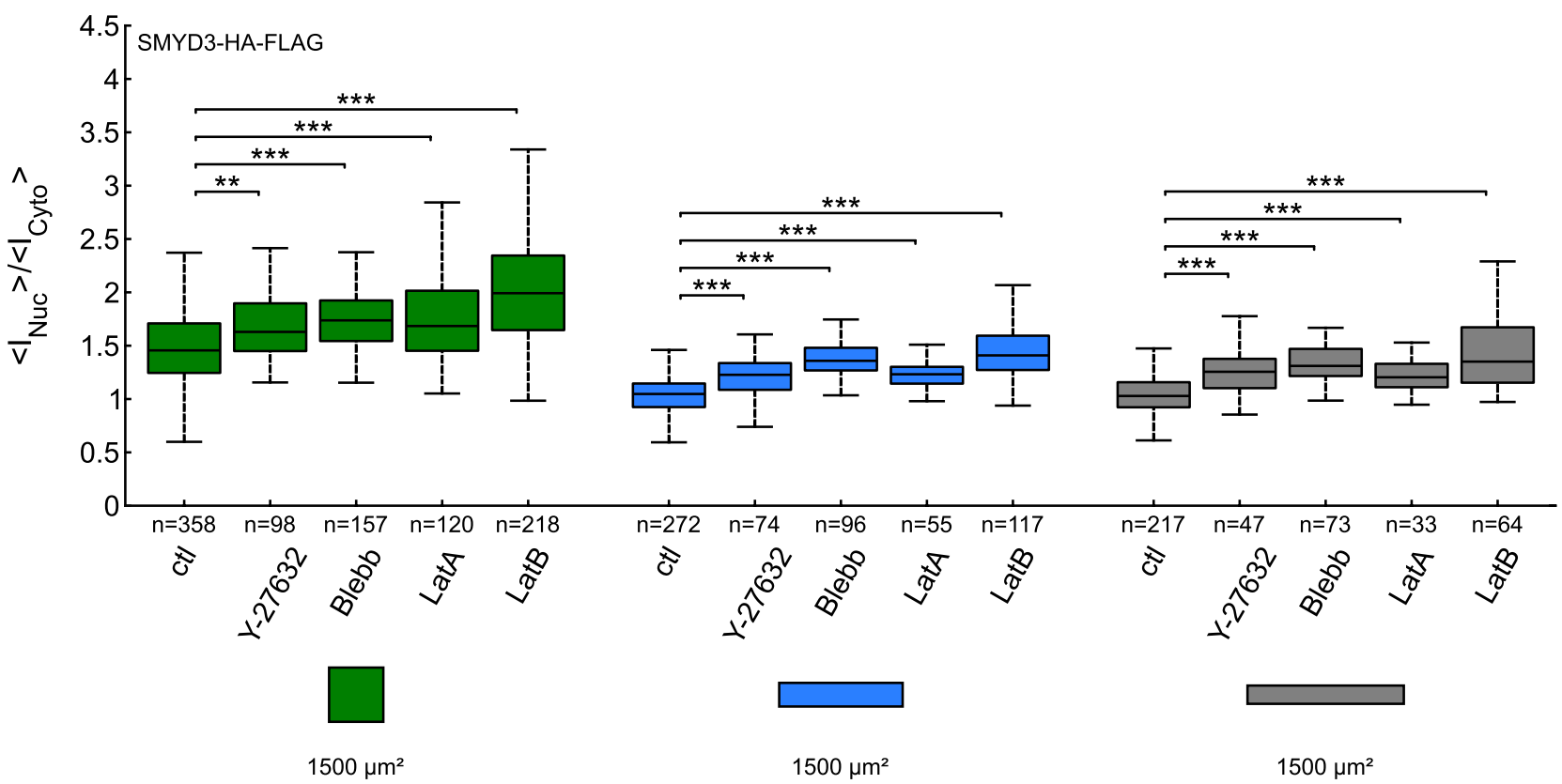

Figure 4. The impact of cytoskeleton disorganisation on SMYD3 cellular distribution. (a) Micrographs of SMYD3-HA-Flag localisation in C2C12 cells treated with drugs targeting actin polymerisation (Latrunculin A or Latrunculin B). (b) Quantification of increased nuclear localization of SMYD3-HA-Flag upon drug treatment of cells plated on non-patterned substrates. A Kruskal-Wallis test was performed. (c) Quantification of increased nuclear localization of SMYD3 upon drug treatment of cells plated on square (green), or rectangle $\left(1: 5\right.$, blue, $1: 8$, grey) patterns. Pattern area $=1800 \mu \mathrm{m}^{2}$. Cells were treated with Y-27632 or blebbistatin (Blebb) or Latrunculin (LatA or LatB). A Kruskal-Wallis test was performed between conditions with identical aspect ratios. $\mathrm{n}=$ number of individual cells measured. ${ }^{\star} p<0.05,{ }^{* *} p<0.01,{ }^{* *} p<0.001, n . s$. not statistically significant.

\section{Methods}

Stamp fabrication. The desired shapes (rectangles and squares) were designed using L-Edit software. A chromium mask was used to create a silicon/SU8 mold with rectangular and square shapes. It was generated by a photolithographic process. The mold was used to carry polydimethylsiloxane (PDMS) overnight at $60{ }^{\circ} \mathrm{C}$, the PDMS layer was peeled off and washed on an ultrasonic bath containing a solution of $70 \%$ of ethanol for $15 \mathrm{~min}$.

Microcontact printing. The structured PDMS stamp was dried with clean dry air and inked for 45 min at room temperature (RT) with a mixture of fibronectin solution from bovine plasma (FN) (Sigma Aldrich) and labeled (cy3.5 Invitrogen labeling kit) fibronectin from human plasma (Roche), the final concentration used for the experiments was $50 \mu \mathrm{g} / \mathrm{ml}$ (containing $2 / 3$ of bovine and $1 / 3$ of human FN). A thin layer of PDMS was exposed to UV/O3 irradiation for $7 \mathrm{~min}$. The FN-coated stamp was washed with phosphate buffer saline (PBS), dried with clean dry air and then brought into contact with the PDMS layer surface for few seconds. The surface 
a

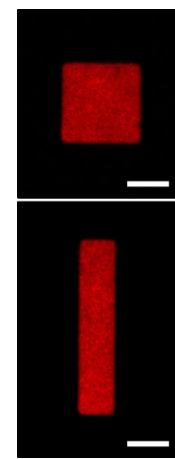

FN

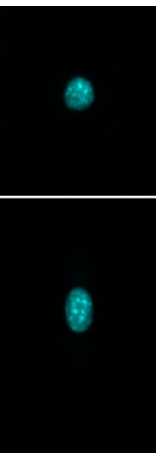

DAPI

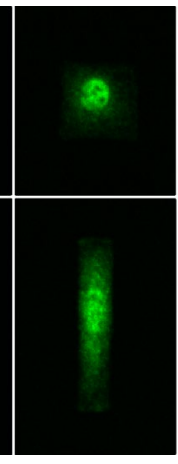

TAZ

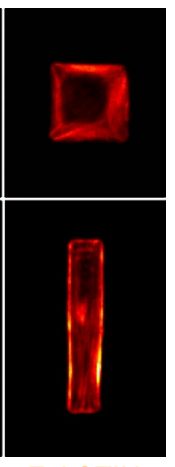

F-ACTIN

b

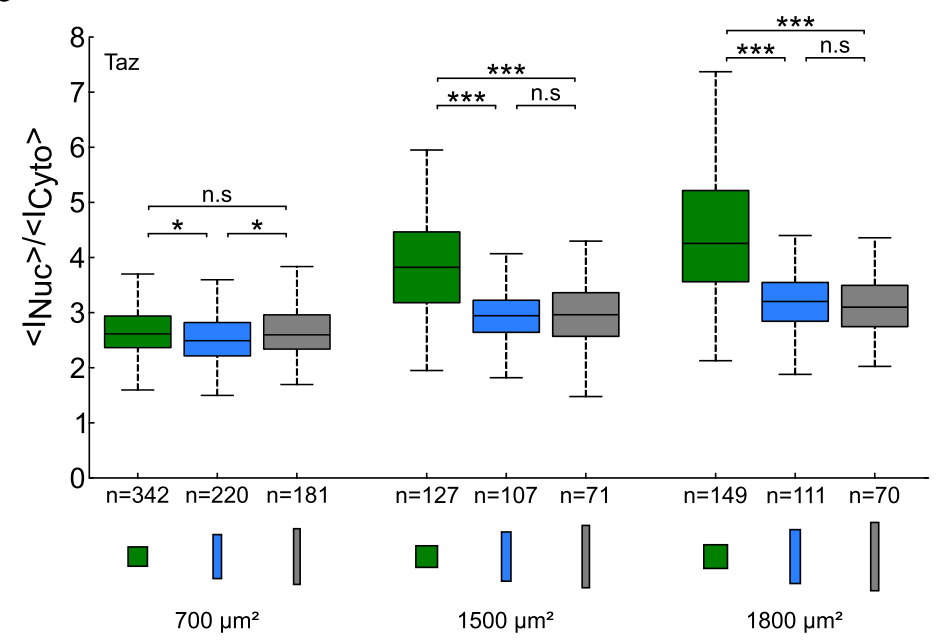

d

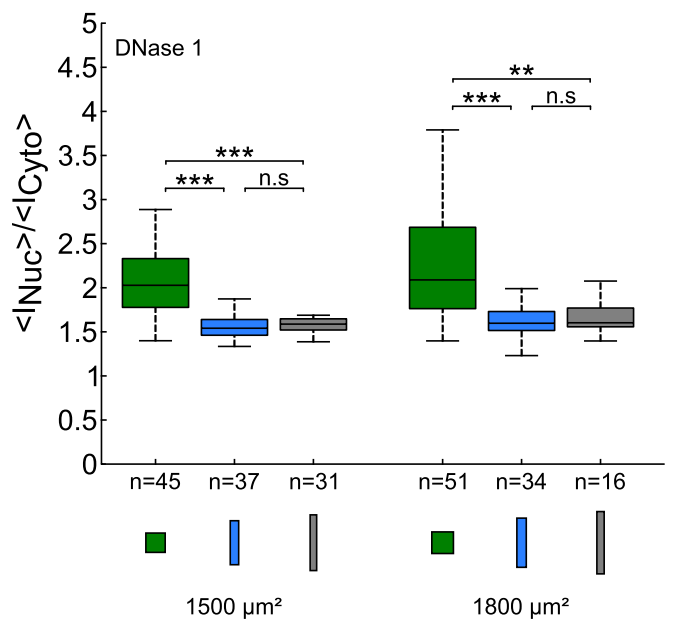

Figure 5. Cell geometry affect the nucleo:cytoplasmic distribution of the YAP/TAZ transcription factor and DNase1 (a) Micrographs of YAP/TAZ (green, anti-TAZ antibody) localisation in C2C12 cells spread on square and rectangles (1:5) micropatterns. The micrographs show the fibronectin (FN) patterning, the nuclear DNA staining (DAPI), YAP/TAZ (green) location and F-actin (SiR-actin). The YAP/TAZ nuclear:cytoplasmic distribution appears different between square and rectangle patterns. Scale bars: $20 \mu \mathrm{m}$. (b) Quantification of the nuclear:cytoplasmic $\left(<\mathrm{I}_{\mathrm{Nuc}}>\left|<\mathrm{I}_{\mathrm{Cyto}}\right\rangle\right)$ distribution ratio for YAP/TAZ over a range of pattern areas (700$\left.1800 \mu \mathrm{m}^{2}\right)$ and geometries: squares (1:1 aspect ratio, green), rectangles (1:5 aspect ratio, blue) and elongated rectangles (1:8 aspect ratio, grey). The median value of the ratio is $13-37 \%$ higher on squares than on rectangles. A Kruskal-Wallis test was performed between conditions with identical areas. An ANOVA test was performed for conditions $700 \mu \mathrm{m}^{2}$. (c) Micrographs of DNase1 (green) localisation in C2C12 cells spread on square (1:1) or rectangle (1:5) micropatterns. The micrographs show the fibronectin (FN) patterning, the nuclear DNA staining (DAPI), DNase 1 (green) localisation and F-actin (SiR-actin). The DNase1 nuclear:cytoplasmic distribution appears different between square and rectangle patterns. Scale bars: $20 \mu \mathrm{m}$. (d) Quantification of the nuclear:cytoplasmic $\left(\left\langle\mathrm{I}_{\mathrm{Nuc}}\right\rangle\left|<\mathrm{I}_{\mathrm{Cyto}}\right\rangle\right)$ distribution ratio for DNase1 over a range of pattern areas (1500$\left.1800 \mu \mathrm{m}^{2}\right)$ and geometries: squares (1:1 aspect ratio, green), rectangles (1:5 aspect ratio, blue) and elongated rectangles (1:8 aspect ratio, grey). The median value of the ratio is about $30 \%$ higher on squares than on rectangle patterns. A Kruskal-Wallis test was performed between conditions with identical areas. $\mathrm{n}=$ number of individual cells measured. ${ }^{\star} \mathrm{p}<0.05,{ }^{* *} p<0.01,{ }^{* *} p<0.001, n . s$. not statistically significant.

was passivated for 45 min with a $0.2 \%$ solution of Pluronic F-127. In most of the experiments, the mold was designed so that all different areas (500-1800 $\mathrm{m}^{2}$ ) and all different shapes (squares, rectangles 1:5 and rectangles 1:8) were on the same stamp, and the cells within a single well faced all the different conditions.

Cells culture and drug treatment. $\mathrm{C} 2 \mathrm{C} 12$ myoblasts cells were cultured in DMEM (high glucose, pyruvate, glutamax) medium (FBS 15\%) and 1\% pen-strep. 5000 cells $/ \mathrm{cm}^{2}$ were plated on the microprinted PDMS layers for $3 \mathrm{~h}$ at $37{ }^{\circ} \mathrm{C}$ in $5 \% \mathrm{CO}_{2}$. C2C12 stably expressing HA-Flag-SMYD3 and C2C12 transfected (Lipofectamine) with eGFP were used. The eGFP plasmid was a kind gift of Alexander Tarakhovsky (Rockefeller 
University, New York) ${ }^{37}$. The chosen drugs were added in the media at the indicated low concentrations to allow spreading and to destabilize the cytoskeleton. The indicated times are measured from the end of the experiment (total time of experiment $3 \mathrm{~h}$ ): Rock inhibitor $10 \mu \mathrm{M} 1 \mathrm{~h}$ (Cell Guidance Systems), Blebbistatin $5 \mu \mathrm{M} 1 \mathrm{~h}$ (Sigma Aldrich), Latrunculin A $0.12 \mu \mathrm{M} 2 \mathrm{~h} 45$ (Sigma Aldrich), Latrunculin B $80 \mathrm{nM} 30 \mathrm{~min}$ (Sigma Aldrich), and LMB 20 nM 2 h (Sigma Aldrich).

Construction of $\mathrm{C} 2 \mathrm{C} 12$ cell line stably expressing HA-Flag-SMYD3. SMYD3 cDNA was amplified by PCR using the following primer pair: (for) cgatgctctcagtgccgcgt and (rev) ggatgctctgatgttggcgtc. cDNA was cloned into the pREV retroviral vector, kindly obtained from the laboratory of Dr S. Ait-Si-Ali (CNRS, UMR7216, Paris). The plasmid contains an epitope tag (3 HA- and 3 Flag-tag) in $5^{\prime}$ of the cloning site and a selection marker. C2C12 myoblasts stably expressing double-tagged Flag-HA-SMYD3 protein were established using retroviral transduction strategy as previously described ${ }^{59}$. C2C12 cells stably integrating pREV-SMYD3 or pREV control vector were sub-cloned to obtain 100\% positive clonal populations. Stable ectopic SMYD3 expression in these clones was validated by Western blot and immunofluorescence analysis, using anti-HA and anti-FLAG antibodies. The overexpression of the exogenous SMYD3-HA-Flag was quantified by Western blot at six exogenous for one endogenous SMYD3 on average (see Figure S1f). We selected two C2C12 positive clonal populations for our further experiments.

Immunochemistry and labeling. After $3 \mathrm{~h}$ in culture on the microprinted PDMS layers, cells were fixed with $4 \%$ PFA at room temperature (RT) for 15 min and then permeabilized with $0.2 \%$ Triton X-100 in PBS for $5 \mathrm{~min}$ at RT and washed 3 times in PBS. The immunochemistry process was performed following these steps: the fixed cells were incubated for 30 min in a blocking solution $(0.2 \%$ tween, $1 \%$ Bovine Serum Albumine BSA, $1 \%$ Foetal Bovine Serum FBS) at RT, then the primary antibody was incubated for 40 min, washed three times in PBS-tween $0.2 \%$, the secondary was incubated for $30 \mathrm{~min}$, washed three times with PBS. The nucleus was then labeled with 4',6-diamidino-2-phenylindole (DAPI) for $30 \mathrm{~min}$ at RT and the actin filaments (F-actin) with SiR-actin (Spirochrome) at $100 \mathrm{nM}$ in PBS overnight at $4{ }^{\circ} \mathrm{C}$. The antibodies used for the immunochemistry were: mouse anti-Flag (Sigma Aldrich M2 F3165) 1/1000, rabbit anti-SMYD3 (Abcam ab187149) 1/300, rabbit anti-TAZ (Cell Signaling Technology 4883) 1/500, mouse anti-YAP (Santa Cruz Biotechnology 101199) 1/250, rabbit anti-Pan tri-methyl lysine (Cell Signaling Technology \#14680) 1/1000, rabbit anti-Pan di-methyl lysine (Cell Signaling Technology \#14117) 1/1000, DNase 1 Alexa Fluor 594 (Life Technologies) 1/1000, Rabbit antiSETDB1 (Santa Cruz Biotechnology sc-66884) 1/200.

Image acquisition. Images were acquired using an inverted microscope (Olympus IX81) equipped with a spinning disk confocal set-up (Andor Revolution XD), a 20X air objective (NA=0.45, LUC PLAN FLN), a $60 \mathrm{X}$ water immersion objective (NA=1.3), four laser diodes $(405 \mathrm{~nm}, 488 \mathrm{~nm}, 561 \mathrm{~nm})$ and band pass filters $(640 \mathrm{~nm} ; 465 / 30 \mathrm{~nm}, 412 / 23 \mathrm{~nm}, 607 / 36 \mathrm{~nm}, 685 / 40 \mathrm{~nm})$ and an Andor iXon EMCCD camera. All the images were acquired with the 20X objective, except that of Fig. 2 and Figure S5, which were acquired with the 60X objective.

Image analysis. Homedesigned codes were written to analyze experimental data, using MATLAB, ImageJ software and Miji plugin (https://imagej.net/Miji). Using this software, we developed an automatized pipeline to segment and measure many parameters such as intensity, shape, aspect ratios, nuclear to cytoplasmic ratios of cells on micropatterns. The Li method was used for segmentation, all image analysis was performed with imageJ. All the parameters (intensities, shape, aspect ratios, etc.) were extracted from imageJ and then analysed with MATLAB. In particular the nuclear:cytoplasmic ratios were calculated from images acquired with the $20 \times$ objective (large depth of focus $\sim 4 \mu \mathrm{m}$ ) as the [Mean intensity per nuclear pixel staining] divided by the [Mean intensity per cytoplasmic pixel staining]). In the particular case of confocal images acquired with a $60 \times$ objective (small depth of focus $\sim 0.5 \mu \mathrm{m}$, images in Fig. 2 ), the calculation of the ratios was made with intensities measured after summing the images of a $z$-stack.

Colocalisation experiments. Images have been acquired using a spinning disk confocal microscope with a $60 \times$ objective (Olympus/Yokogawa/Andor). For each cell, a characteristic plane (XY) of each channel has been used to plot the intensity of Kme3 (or Kme2) vs SMYD3 intensity. This has been done for the nucleus and the cytoplasm separately. The estimation of the correlation of the intensities has been done using the Pearson's correlation coefficient $\left(\rho_{\text {Pearson }}=\frac{\operatorname{cov}\left(I_{S M Y D 3}, I_{\text {Kme3 }}\right)}{\sigma_{S M Y D 3} \sigma_{K m e 3}}\right)$.

FRAP experiments. Cells transfected with SMYD3-eGFP were plated on squares and rectangles micropatterns (area of $1500 \mu \mathrm{m}^{2}$ ). To measure the nuclear import (export), we bleached the whole nucleus (cytoplasm) and we measured the fluorescence recovery after the photobleaching in the nucleus. Then, the experimental curves have been fitted using exponential functions.

Statistical analysis. Different statistical analyses were performed depending on the number $\mathrm{N}$ of distributions and on the number $n$ of measurements in each distribution. When $n \geq 30$, the significance was tested using a Student test for the comparison between $\mathrm{N}=2$ distributions and a one-way Anova test (Tukey-Kramer posthoc test) when $\mathrm{N}>2$. When $\mathrm{n}<30$, the normality of the distributions was first tested using an Anderson-Darling test. For normal distributions, the tests were the same as when $n \geq 30$. If the distributions were not normal, the significance was tested using a Wilcoxon rank sum test for the comparison between $\mathrm{N}=2$ distributions and a 
Kruskal-Wallis test (Dunn and Sidak posthoc test) when $\mathrm{N}>2$. In the case of unequal variance (Levene's test) between the distributions the Kruskal-Wallis test was used instead of a one-way ANOVA. The statistical analysis was performed using Matlab. For all the analyses, all the cells from different repeats of the same experimental conditions were pooled. The numbers of experiments and repeats are listed in Table S1.

Received: 11 February 2020; Accepted: 25 September 2020

Published online: 26 November 2020

\section{References}

1. Chen, C. S., Mrksich, M., Huang, S., Whitesides, G. M. \& Ingber, D. E. Geometric control of cell life and death. Science 276, 1425-1428 (1997).

2. Engler, A. J., Sen, S., Sweeney, H. L. \& Discher, D. E. Matrix elasticity directs stem cell lineage specification. Cell 126, 677-689 (2006).

3. Icard-Arcizet, D., Cardoso, O., Richert, A. \& Hénon, S. Cell stiffening in response to external stress is correlated to actin recruitment. Biophys. J. 94, 2906-2913 (2008).

4. Mitrossilis, D. et al. Real-time single-cell response to stiffness. Proc. Natl. Acad. Sci. 107, 16518-16523 (2010).

5. Lo, C. M., Wang, H. B., Dembo, M. \& Wang, Y. L. Cell movement is guided by the rigidity of the substrate. Biophys. J. 79, 144-152 (2000).

6. Engler, A. J. et al. Myotubes differentiate optimally on substrates with tissue-like stiffness: Pathological implications for soft or stiff microenvironments. J. Cell Biol. 166, 877-887 (2004).

7. Kilian, K. A., Bugarija, B., Lahn, B. T. \& Mrksich, M. Geometric cues for directing the differentiation of mesenchymal stem cells. Proc. Natl. Acad. Sci. USA 107, 4872-4877 (2010).

8. Dupont, S. et al. Role of YAP/TAZ in mechanotransduction. Nature 474, 179-183 (2011).

9. Montel, L., Sotiropoulos, A. \& Hénon, S. The nature and intensity of mechanical stimulation drive different dynamics of MRTF-A nuclear redistribution after actin remodeling in myoblasts. PLoS ONE 14, 1-22 (2019).

10. Kouzarides, T. Chromatin modifications and their function. Cell 128, 693-705 (2007).

11. Klose, R. J. \& Zhang, Y. Regulation of histone methylation by demethylimination and demethylation. Nat. Rev. Mol. Cell Biol. 8, 307-318 (2007).

12. Greer, E. L. \& Shi, Y. Histone methylation: A dynamic mark in health, disease and inheritance. Nat. Rev. Genet. 13, 343-357 (2012).

13. Bannister, A. J. \& Kouzarides, T. Regulation of chromatin by histone modifications. Cell Res. 21, 381-395 (2011).

14. Kouzarides, T. Histone methylation in transcriptional control. Curr. Opin. Genet. Dev. 12, 198-209 (2002).

15. Cheung, P. \& Lau, P. Epigenetic regulation by histone methylation and histone variants. Mol. Endocrinol. 19, 563-573 (2005).

16. Jin, W., Peng, J. \& Jiang, S. The epigenetic regulation of embryonic myogenesis and adult muscle regeneration by histone methylation modification. Biochem. Biophys. Rep. 6, 209-219 (2016).

17. Husmann, D. \& Gozani, O. Histone lysine methyltransferases in biology and disease. Nat. Struct. Mol. Biol. 26, 880-889 (2019).

18. Dillon, S. C., Zhang, X., Trievel, R. C. \& Cheng, X. The SET-domain protein superfamily: Protein lysine methyltransferases. Genome Biol. 6, 227 (2005).

19. Shi, X. et al. ING2 PHD domain links histone H3 lysine 4 methylation to active gene repression. Nature 442, $96-99$ (2006).

20. Chuikov, S. et al. Regulation of p53 activity through lysine methylation. Nature 432, 353-360 (2004).

21. Oh, H. et al. Yorkie promotes transcription by recruiting a histone methyltransferase complex. Cell Rep. 8, 449-459 (2014).

22. Zhao, L. et al. JMJD2B promotes epithelial-mesenchymal transition by cooperating with $\beta$-catenin and enhances gastric cancer metastasis. Clin. Cancer Res. 19, 6419-6429 (2013).

23. Carlson, S. M. \& Gozani, O. Nonhistone lysine methylation in the regulation of cancer. Cold Spring Harb. Perspect. Med. 6, a026435 (2016).

24. Mazur, P. K. et al. SMYD3 links lysine methylation of MAP3K2 to Ras-driven cancer. Nature 510, 283-287 (2014).

25. Beyer, S. et al. Canonical Wnt signalling regulates nuclear export of Setdb1 during skeletal muscle terminal differentiation. Cell Discov. 2, 16037 (2016).

26. Proserpio, V., Fittipaldi, R., Ryall, J. G., Sartorelli, V. \& Caretti, G. The methyltransferase SMYD3 mediates the recruitment of transcriptional cofactors at the myostatin and c-Met genes and regulates skeletal muscle atrophy. Genes Dev. 27, 1299-1312 (2013).

27. Cock-Rada, A. M. et al. SMYD3 promotes cancer invasion by epigenetic upregulation of the metalloproteinase MMP-9. Cancer Res. 72, 810-820 (2012).

28. Peserico, A. et al. A SMYD3 Small-Molecule Inhibitor Impairing Cancer Cell Growth. J. Cell. Physiol. 230, 2447-2460 (2015).

29. Hamamoto, R. et al. Enhanced SMYD3 expression is essential for the growth of breast cancer cells. Cancer Sci. 97, 113-118 (2006).

30. Sarris, M. E., Moulos, P., Haroniti, A., Giakountis, A. \& Talianidis, I. Smyd3 is a transcriptional potentiator of multiple cancerpromoting genes and required for liver and colon cancer development. Cancer Cell 29, 354-366 (2016).

31. Hamamoto, R. et al. SMYD3 encodes a histone methyltransferase involved in the proliferation of cancer cells. Nat. Cell Biol. 6, 731-740 (2004).

32. van Aller, G. S. et al. Smyd 3 regulates cancer cell phenotypes and catalyzes histone H4 lysine 5 methylation. Epigenetics 7, 340-343 (2012).

33. Tsai, C. H. et al. SMYD3-mediated H2AZ1 methylation promotes cell cycle and cancer proliferation. Cancer Res. 76, 6043-6053 (2016).

34. Kunizaki, M. et al. The lysine 831 of vascular endothelial growth factor receptor 1 is a novel target of methylation by SMYD3. Cancer Res. 67, 10759-10765 (2007).

35. Codato, R. et al. The SMYD3 methyltransferase promotes myogenesis by activating the myogenin regulatory network. Sci. Rep. $\mathbf{9}$, 17298 (2019).

36. Fujii, T., Tsunesumi, S., Yamaguchi, K., Watanabe, S. \& Furukawa, Y. Smyd3 is required for the development of cardiac and skeletal muscle in zebrafish. PLoS ONE 6, e23491 (2011).

37. Donlin, L. T. et al. Smyd2 controls cytoplasmic lysine methylation of Hsp90 and myofilament organization. Genes Dev. 26, 114-119 (2012).

38. Bruyère, C. et al. Actomyosin contractility scales with myoblast elongation and enhances differentiation through YAP nuclear export. Sci. Rep. 9, 15565 (2019).

39. Bentzinger, C. F., Wang, Y. X. \& Rudnicki, M. A. Building muscle: molecular regulation of myogenesis. Cold Spring Harb. Perspect. Biol. 4, a008342 (2012).

40. Asp, P. et al. Genome-wide remodeling of the epigenetic landscape during myogenic differentiation. Proc. Natl. Acad. Sci. 108, E149-E158 (2011). 
41. Nagandla, H. et al. Defective myogenesis in the absence of the muscle-speci fi c lysine methyltransferase SMYD1. Dev. Biol. 410, 86-97 (2016).

42. Wei, X., Henke, V. G., Strübing, C., Brown, E. B. \& Clapham, D. E. Real-time imaging of nuclear permeation by EGFP in single intact cells. Biophys. J. 84, 1317-1327 (2003).

43. Dai, B. et al. SET and MYND domain-containing protein 3 is overexpressed in human glioma and contributes to tumorigenicity. Oncol. Rep. 34, 2722-2730 (2015).

44. Brown, M. A. et al. C-terminal domain of SMYD3 serves as a unique HSP90-regulated motif in oncogenesis. Oncotarget 6, 4005-4019 (2015).

45. Stade, K., Ford, C. S., Guthrie, C. \& Weis, K. Exportin 1 (Crmlp) is an essential nuclear export factor. Cell 90, 1041-1050 (1997).

46. Fornerod, M., Ohno, M., Yoshida, M. \& Mattaj, I. W. CRM1 is an export receptor for leucine-rich nuclear export signals. Cell 90, 1051-1060 (1997).

47. Kudo, N. et al. Leptomycin B inactivates CRM1/exportin 1 by covalent modification at a cysteine residue in the central conserved region. Proc. Natl. Acad. Sci. USA 96, 9112-9117 (1999).

48. Charrier, E. E. et al. Desmin mutation in the C-terminal domain impairs traction force generation in myoblasts. Biophys. J. 110, $470-480$ (2016).

49. Kim, D. H., Chambliss, A. B. \& Wirtz, D. The multi-faceted role of the actin cap in cellular mechanosensation and mechanotransduction. Soft Matter 9, 5516-5523 (2013).

50. Versaevel, M. et al. Super-resolution microscopy reveals LINC complex recruitment at nuclear indentation sites. Sci. Rep. 4, 7362 (2014).

51. Percipalle, P. \& Visa, N. Molecular functions of nuclear actin in transcription. J. Cell Biol. 172, 967-971 (2006).

52. Sero, J. E. et al. Cell shape and the microenvironment regulate nuclear translocation of NF-kB in breast epithelial and tumor cells. Mol. Syst. Biol. 11, 790 (2015).

53. Azzolin, L. et al. YAP/TAZ incorporation in the $\beta$-catenin destruction complex orchestrates the Wnt response. Cell 158, 157-170 (2014).

54. Sorrentino, G. et al. Metabolic control of YAP and TAZ by the mevalonate pathway. Nat. Cell Biol. 16, 357-366 (2014).

55. Gnimassou, O., Francaux, M. \& Deldicque, L. Hippo pathway and skeletal muscle mass regulation in mammals: A controversial relationship. Front. Physiol. 8, 1-6 (2017).

56. Le, H. Q. et al. Mechanical regulation of transcription controls Polycomb-mediated gene silencing during lineage commitment. Nat. Cell Biol. 18, 864-875 (2016).

57. Jain, N., Iyer, K. V., Kumar, A. \& Shivashankar, G. V. Cell geometric constraints induce modular gene-expression patterns via redistribution of HDAC3 regulated by actomyosin contractility. Proc. Natl. Acad. Sci. USA 110, 11349-11354 (2013).

58. Lachowski, D. et al. Matrix stiffness modulates the activity of MMP-9 and TIMP-1 in hepatic stellate cells to perpetuate fibrosis. Sci. Rep. 9, 1-9 (2019).

59. Joliot, V. et al. The SWI/SNF subunit/tumor suppressor BAF47/INI1 is essential in cell cycle arrest upon skeletal muscle terminal differentiation. PLoS One 9, e10885 (2014).

\section{Acknowledgements}

We thank members of the Hénon and Weitzman laboratories and members of the UMR7216 Epigenetics and Cell Fate for discussions and advice on this study. We acknowledge the ImagoSeine core facility of the Institut Jacques Monod (member of the France BioImaging, ANR-10-INBS-04). This work was supported by the LabEx "Who Am I?" \#ANR-11-LABX-0071 and the Université de Paris IdEx \#ANR-18-IDEX-0001 funded by the French Government through its "Investments for the Future" program. Additional funding from AFMTELETHON (\#16146), and the Fondation ARC pour la Recherche sur le Cancer (ARC no. 155029). JBW is a Senior member of the Institut Universitaire de France (IUF) and SM is a Junior member (2012ND 3369).

\section{Author contributions}

S.H. and J.B.W. developed the conceptual framework, sought funding and provided overall supervision. D.P. and A.R. performed the experiments. D.P. performed all the quantitative analysis of the results. D.P., S.M., S.H. and J.B.W. designed the study, analysed the findings and wrote the manuscript.

\section{Competing interests}

The authors declare no competing interests.

\section{Additional information}

Supplementary information is available for this paper at https://doi.org/10.1038/s41598-020-75833-9.

Correspondence and requests for materials should be addressed to S.H. or J.B.W.

Reprints and permissions information is available at www.nature.com/reprints.

Publisher's note Springer Nature remains neutral with regard to jurisdictional claims in published maps and institutional affiliations.

Open Access This article is licensed under a Creative Commons Attribution 4.0 International License, which permits use, sharing, adaptation, distribution and reproduction in any medium or format, as long as you give appropriate credit to the original author(s) and the source, provide a link to the Creative Commons licence, and indicate if changes were made. The images or other third party material in this article are included in the article's Creative Commons licence, unless indicated otherwise in a credit line to the material. If material is not included in the article's Creative Commons licence and your intended use is not permitted by statutory regulation or exceeds the permitted use, you will need to obtain permission directly from the copyright holder. To view a copy of this licence, visit http://creativecommons.org/licenses/by/4.0/.

(c) The Author(s) 2020 